\title{
GENETIC STUDIES ON THE EFFECTS OF DROUGHT TOLERANCE AND EARLINESS ON GRAIN YIELD IN DURUM WHEAT HAVING DIFFERENT THERMAL RESPONSES
}

\author{
ABDEL NOUR, NADYA A. R. ${ }^{1}$ and HAYAM S.A. FATEH ${ }^{2}$
}

1. Wheat Research Dep., Field Crops Research Institute, ARC, Giza, Egypt.

2. Lab. for Design and Statistical Analysis Research, ARC.

(Manuscript received 22 August 2011)

\begin{abstract}
A diallel cross involving six durum wheat genotypes was evaluated to determine the genetic behavior of earliness components as well as yield and its components in durum wheat under irrigation and drought conditions. In addition, to drought susceptibility index, thermal unit and reduction percentage were calculated. The resultant hybrids along with their parents were evaluated in two experiments. The first experiment (stress) was irrigated once (70 days after planting while the second one (normal) was irrigated four times at Sids the experiment Station, Agriculture Research Centre.

Genotypes mean squares were significant for most studied characters in the two experiments except for heading under normal experiment. GCA variance values were higher two or more times than the SCA variance one for most studied characters in both experiments, suggesting the predominant of additive and additive $x$ additive gene action in controlling these characters. Parent $P_{1}$ (Sohage1) could be considered as a good combiner for early heading and maturity under stress for days and thermal units; $P_{2}$ (Sohage3) could be considered as a good combiner for early maturity under stress and normal of days and only under stress of thermal unit, while $\mathrm{P}_{3}$ (Beni Swief 6) could be considered as a good combiner for (early maturity under normal irrigation). Mareover, Parent $\mathrm{P}_{4}$ ( Portoroco) could be considered as a good combiner for grain yield /plant and kernel weight under stress and normal irrigation and susceptibility index for No. of spikes/ plant meanwhile, parent $P_{5}$ (Quadrato) is a good combiner for grain yield/ plant, grain filling rate and No. of spikes/ plant of the two experiments and susceptibility index for both No. of kernels/ spike and kernel weight but parent $\mathrm{P}_{6}$ (Beni Swief1) considered a good combiner for grain yield/ plant and grain filling rate under two stress and normal irrigation and also early maturing of No. of days under only irrigation only. The best SCA values were detected for cross $\mathrm{P}_{1} \times \mathrm{P}_{4}$ for the earliness of maturity (No. of days and thermal units) under stress water and the reduction percentage for number of days and thermal units of normal to stress conditions, grain filling rate, susceptibility index for No. of spikes/ plant, No. of kernels/ spike and kernel weight, and grain yield/ plant under both stress and normal conditions, crosses $\mathrm{P}_{1} \times \mathrm{P}_{6}, \mathrm{P}_{2} \times \mathrm{P}_{6}, \mathrm{P}_{3} \times \mathrm{P}_{4}$ and $P_{3} \times P_{5}$ gave the best SCA effects for grain yield/ plant under both stress and normal conditions.
\end{abstract}

Key words: Wheat, Drought, Combining ability, Susceptibility index, early maturing, Earliness characters. 


\section{INTRODUCTION}

The new Egyptian policy for wheat production is to increase the area cultivated to durum wheat (Triticum turigidum L. var. durum) in Upper Egypt because of its high tolerance to the prevalent hot weat and the need for macaroni industry.

The Egyptian wheat cultivars have relatively narrow genetic background. Selection among these cultivars for increasing grain yield and its components would not be very effective. Hybridization between the Egyptian wheat cultivars and exotic materials was carried out to increase the genetic variability.

The ultimate goal of wheat breeder is to develop new genotypes characterized by high yield potentiality and tolerance to stress conditions such as drought. To achieve this target some important genetic information are required about drought susceptibility indices for yield and yield components. Such genetic information directs the breeding program towards the use of selection if the additive gene action is predominant or to exploit heterosis if non additive gene action was prevailed in controlling the traits of interest.

Heading time is affected by complex interactions of temperature and photoperiod (Masle et. al. 1989). The three components of heading time are vernalization requirement, photoperiod response, and intrinsic- earliness. These components may act individually or in combination to achieve different adaptation strategies according to Kato and Yokoyama (1992)

Developing early - maturing wheat is important for increasing cultivated area of wheat through planting in the areas designated for growing cotton in summer season. Early maturing cultivars are also preferable to escape diseases, pests, drought, heat and other stress injuries that occur at the end of the growing season Clarke et. al. 1984.Therefore,. Breeding early- maturing cultivars is an important objective in most wheat breeding programs.

Losses in grain yield and its components of wheat due to water stress were recorded by many investigates (Abul- Naas et. al. 2000, Abdel- Nour, Nadya 2005 and Abdel - Nour, Nadya and Manal A. Hassam 2009). They reported that water stress can be considered as major a biotic stress affecting wheat yield. Yield lasses due to certain stresses may be minimized in early - maturing cultivars, since they would escape such stresses that might occur late in season ((larke et. al. 1984). However, Fischer and Maurer (1978) reported that early - maturing cultivars were more drought tolerant than late ones. A better understanding of the inheritance and type of gene action for earliness and grain filling traits would help wheat breeders to incrand stabilize grain yield. 
Regarding drought susceptibility index, Abul- Naas et. al. (2000) and Abdel Nour (2005) reported that additive gene action was predominant in the inheritance of total plant weight and straw yield, while non additive gene action was important in controlling number of spikes/ plant, kernel weight and grain yield.

\section{The aim of the present work was to:}

1- study water stress effects on earliness and grain filling traits as well as grain yield and its components for some durum wheat genotypes. 2- Determine some early maturing and high yielding genotypes under drought stress conditions.

3- Study general and specific combining ability for yield and its components and earliness components under normal and drought conditions as well as drought susceptibility index and thermal units in durum wheat. It is hoped that this study may help wheat breeders in developing new genotypes with high yield potentiality and tolerant to drought stress.

\section{MATERIALS AND METHODS}

This study was conducted during the two successive seasons 2008/2009 and 2009/2010. Six widely diversed durum wheat genotypes were used. Four local cultivars; Sohage 1, Sohage 3, Beni Swef 6 and Beni Swef 1 and two exotic cultivars ( Portoroco and Quadrato) introduced from Italy, representing a wide range of diversity for several agronomic characters and drought tolerance as well as thermal units for heading and maturity were used for this study.

The pedigree and origin of these parental materials are presented in Table (1).

Table 1. Name, pedigree and origin of Six parental durum wheat cultivars.

\begin{tabular}{|l|l|l|l|}
\hline No. & Name & Pedigree & Origin \\
\hline 1 & Sohage1 & GDOVZ46913/JO"S"// 61.130-LDS & Egypt \\
\hline 2 & Sohage3 & MEXI"S"/ MGHA/ 51792// DURUM6 & Egypt \\
\hline 3 & Beni Swief 6 & BOOMER-21/ BUSCA-3 & Egypt \\
\hline 4 & Portoroco & & Italy \\
\hline 5 & Quadrato & & Italy \\
\hline 6 & Beni Swief 1 & Jo "S" / AA "S" // Fg "s" & Egypt \\
\hline
\end{tabular}

This study was carried out at El- Giza Research Station during 2008/ 2009 season. The parental genotypes were sown at various dates to overcome the 
differences in time of heading and secure enough time for making crosses and hence getting more hybrid seeds for evaluation. Parents were crossed in all possible combinations excluding reciprocals to obtain total of $15 \mathrm{~F} 1$ hybrids.

In 2009/2010 season, the six parents along with their single crosses (15 crosses) were sown at Sids Research Station, Beni Swef Governorate ARC, in two adjacent experiments. The first experiment (Stress) was irrigated once (70 days after planting irrigation).Meanwhile, the second experiment (non stress or normal) was irrigated four times after planting irrigation. A border of fifteen meter was set between the two experiments. Each experiment was arranged in a randomized complete block design with three replications. Each experimental unit consisted of one row of three meter long, with single plants spaced $20 \mathrm{~cm}$ and $30 \mathrm{~cm}$ between rows. The proper cultural practices were applied as recommended for wheat production in both experiments. The amount of total rainfall during the second growing season was recorded in Table (2). Water table was estimated ( $150 \mathrm{~cm}$.).

Table 2. Monthly average of total rainfall at Beni Swef governorate during 2009/2010 winter season.

\begin{tabular}{|l|l|l|l|l|l|l|l|}
\hline Month & Nov.2009 & Dec.2009 & Jon.2010 & Feb. 2010 & Mor.2010 & Apr.2010 & May.2010 \\
\hline $\begin{array}{l}\text { Rainfall } \\
\text { mm/month }\end{array}$ & 0.15 & 0.30 & 0.20 & 0.24 & 0.04 & 0.00 & 0.00 \\
\hline
\end{tabular}

Observation and measurements were recorded in both experiments as mean value of ten individual guarded plants from each row. The studied characters were earliness components', number of days to heading, number of days to maturity, grain filling period (that is number of days from heading to maturity) and grain filling rate (the grain yield divided by grain filling period).In addition, number of days to heading and number of days to maturity also were expressed as thermal unit time (TTi). Thermal time was calculated as the accumulation of degree- days, (TTi) considering that temperature changed linearly during the day between maximum and minimum temperature fallowing a triangular function:

$T T i=((T \max i+T \min i) / 2)-T b$

$\mathrm{Tb}=\mathrm{O}^{\circ} \mathrm{C}$ 
Where $\mathrm{T} \max \mathrm{i}$ and $\mathrm{T} \min \mathrm{i}$ are the maximum and minimum daily air temperature on the $i^{\text {th }}$ day and $\mathrm{Tb}$ is the base temperature below which the rate of development is assumed to be zero (Gomez- Macpherson and Richard, 1997).

Table 3. Average of monthly temperature at Bani Suef governorate during 2009/2010 wheat growing season.

\begin{tabular}{|l|l|l|}
\hline Month & Period & Average temperature \\
\hline November & $21-30$ & 18.5 \\
\hline December & $1-10$ & 19.2 \\
& 11.20 & 17.5 \\
& $21-31$ & 15.1 \\
\hline January & $1-10$ & 13.8 \\
& 11.20 & 15.9 \\
\hline \multirow{2}{*}{ February } & $21-31$ & 16.4 \\
\hline \multirow{2}{*}{ March } & $1-10$ & 17.4 \\
& $11-20$ & 16.7 \\
& $21-28$ & 14.9 \\
\hline \multirow{2}{*}{ April } & $1-10$ & 16.7 \\
& 11.20 & 17.0 \\
& $21-31$ & 16.9 \\
\hline May & $1-10$ & 21.4 \\
& 11.20 & 22.9 \\
& $21-30$ & 22.6 \\
\hline & $1-10$ & 23.3 \\
& 11.20 & 26.3 \\
& $21-31$ & 26.4 \\
\hline
\end{tabular}

Average $=($ Maximum + Minimum $) / 2$

Measurements were recorded under stress and normal experments for number of spikes/ plant, number of kernels/ spike, 100-kernel weight and grain yield/ plant.

The susceptibility index (S.I) was used as a measure for drought tolerance in terms of minimization of the reduction in grain yield or yield components caused by unfavorable versus favorable environments. (S.I) was calculated for each genotype according to the formula of Fischer and Maurer (1978).

$S . I=\left(1-Y_{S} / Y_{N}\right) / D$ 
Where:

S.I = an index of drought susceptibility

$Y_{S}=$ yield or yield components from stress experiments of a genotype

$\mathrm{Y}_{\mathrm{N}}=$ yield or yield components from normal irrigation experiment of a genotype.

$D=$ drought intensity $=1$ - $\left(\right.$ mean $Y_{S}$ of all genotypes $/$ mean $Y_{N}$ of all genotypes)

Analysis of variance was performed for all studied characters in stress and normal irrigation experiments as well as susceptibility index according to Steel and Torrie (1980). General and specific combining abilities were estimated according to Griffin (1956) as method 2 model 1.

\section{RESULTS AND DISCUSSION}

Analysis of variance and mean performance:

Analysis of variance for all the studied characters in stress and non stress experiments as well as drought susceptibility index and thermal unit is presented in Table ( $4 a$ and $b$ ). Results indicated that mean squares due to genotypes were significant for all characters except for number of days and thermal units of heading in normal experiment, indicating a wide range of diversity for the studied materials. Mean squares due to both parents and crosses were significant for most characters in both experiments including reduction percentage, thermal units and susceptibility index.

Mean performance for parents and their hybrids are presented in Table (5). Under stress condition, parent $P_{1}$ expressed the lowest mean for number of days and thermal units of maturity, while, parent $P_{5}$ had the highest mean value for grain filling rate, and parent $\mathrm{P}_{5}$ exhibited the most desirable values for the number of spikes/ plant, number of kernels/ spike and grain yield / plant. On the other hands normal irrigation experiment, $\mathrm{P}_{1}$ and $\mathrm{P}_{2}$ expressed the lowest mean value favorable for number of days and thermal units of maturity while parent ( $P$ 6) recorded the highest reduction. For the thermal unit of maturity, the highest desirable mean values were recorded by parent $\mathrm{P}_{5}$ for number of spike/plant, number of kernels / spike and grain yield / plant and $\mathrm{P}_{4}$ for grain weight. For drought susceptibility index, the most desirable mean value were detected by parent $\mathrm{P}_{2}$ for number of spikes/ plant and $\mathrm{P}_{5}$ for number of kernels / spike, grain weight and grain yield / plant. Regarding hybrid mean performance, lowest desirable values in stress experiment were recorded by cross combination $\mathrm{P}_{1} \times \mathrm{P}_{2}$ for maturity (days and thermal units), Meanwhil $\mathrm{P}_{1} \times \mathrm{P}_{4}$ 
had the highest value for grain filling rate, $\mathrm{P}_{3} \times \mathrm{P}_{5}$ for the number of spikes / plant and grain yield / plant, $\mathrm{P}_{1} \times \mathrm{P}_{6}$ for number of kernels / spikes and $\mathrm{P}_{5} \times \mathrm{P}_{6}$ for grain weight. Such results indicate that these cross combinations are promising and prospective in drought condition. Under normal irrigation, the best hybrids were $\mathrm{P}_{1} \times \mathrm{P}$ 5, $\mathrm{P}_{1} \times \mathrm{P}_{6}, \mathrm{P}_{3} \times \mathrm{P}_{4}$ and $\mathrm{P}_{4} \times \mathrm{P}_{6}$ which had the lowest values (favorable) for the maturity (days and thermal units). Moreover, $\mathrm{P}_{1} \times \mathrm{p}_{6}$ had the highest value for grain filling rate; $\mathrm{P}_{3} \times \mathrm{P}_{5}$ for the number of spikes / plant, $\mathrm{P}_{1} \times \mathrm{P}_{2}$ for number of kernels / spike, $\mathrm{P}_{4} \times \mathrm{P}_{6}$ for the 100 - grain weight and $\mathrm{P}_{1} \times \mathrm{P}_{6}$ for the grain yield / plant. The most desirable hybrids for reduction were recorded for by the cresses $\left(\mathrm{P}_{1} \times \mathrm{P}_{4}\right.$ for number of days and $\mathrm{P}_{1} \times \mathrm{P}_{5}$ for thermal units) for maturity, while $\mathrm{P}_{1} \times \mathrm{P}_{4}$ had less reduction (favorable) for grain filling rate. The most desirable hybrids for drought susceptibility index were by the cross $\mathrm{P}_{3} \times \mathrm{P}_{5}$ for number of spikes / plant, $\mathrm{P}_{2} \times \mathrm{P}_{6}$ for number of kernels / spike, and $\mathrm{P}_{2} \times \mathrm{P}_{5}$ for 100 - grain weight and grain yield / plant.

From these results, it could be concluded that the three crosses $\mathrm{P}_{1} \times \mathrm{P}_{6}$, $\mathrm{P}_{4} \times \mathrm{P}_{6}$ and $\mathrm{P}_{3} \times \mathrm{P}_{5}$ were the best among the studied hybrids since they expressed the most desirable values for early maturing and most characters under stress and normal irrigation conditions. However, $\mathrm{P}_{3} \times \mathrm{P}_{4}$ have also desirable values under susceptibility index.In this connection several investigators reported that there was a wide range of response to drought resistance in wheat genotypes. Abul - Naas et. al. (2000), Ammar (2003), Abdel - Nour, Nadya (2005), Menshawy (2005), Menshawy (2007) and Abdel - Nour, Nadya and Hassan (2009).

\section{Combining ability analysis}

Analysis of variance for combining ability in stress and normal experiments as well as thermal units, reduction and drought susceptibility index are presented in Table ( $4 \mathrm{a}$ and $\mathrm{b}$ ). Mean squares associated with general (GCA) and specific (SCA) combining abilities were significant for most studied characters under both experiments and also thermal units, reduction and drought susceptibility index. High GCA / SCA ratios which largely exceeded the unity were detected for most of traits under study in both experiments. Such results indicated that the additive and additive $x$ additive of gene actions are the two main types responsible for the inheritance of these characters. The importance of additive genetic variance for durum wheat grain yield susceptibility index and its components as well as drought resistance was previously reported by khalifa et. al. (1998), Abul -Naas et. al. (2000), Abdel - Nour, Nadya (2005) Menshawy (2005), and Abdel- Nour, Nadya and Hassan (2009). 
Table 4a: Mean squares analysis for the earliness components under water stress and normal irrigated conditions.

\begin{tabular}{|c|c|c|c|c|c|c|c|c|c|c|}
\hline \multirow{3}{*}{ S.O.V } & \multirow{3}{*}{ d.f } & \multicolumn{6}{|c|}{ Heading } & \multicolumn{3}{|c|}{ Maturity } \\
\hline & & \multicolumn{3}{|c|}{ Days } & \multicolumn{3}{|c|}{ Thermal units } & \multicolumn{3}{|c|}{ Days } \\
\hline & & Stress & Normal & Red\% & Stress & Normal & Red\% & Stress & Normal & Red\% \\
\hline Rep & 2 & 23.467 & 19.875 & 0.014 & 5284 & 5176 & 0.012 & 13.312 & 4.375 & 0.039 \\
\hline Genotypes & 20 & $6.031^{*}$ & 5.319 & $5.785^{* *}$ & $1415.2^{*}$ & 1392.8 & $5.437 * *$ & $39.55^{* *}$ & $28.738^{* *}$ & $6.751^{* *}$ \\
\hline $\begin{array}{l}\text { Parent } \\
(\mathrm{P})\end{array}$ & 5 & $5.6^{*}$ & 6.134 & $5.766^{* *}$ & 1314.4* & 1586.4 & $5.147^{* *}$ & $92.988 * *$ & $70.319 * *$ & $8.016^{* *}$ \\
\hline $\begin{array}{c}\text { Crosses } \\
\text { (c) }\end{array}$ & 14 & $6.313^{*}$ & 5.368 & $6.12 * *$ & 1482.86* & 1410.29 & $5.84 * *$ & $14.589 * *$ & $6.205^{*}$ & $6.781 * *$ \\
\hline prsc & 1 & 4.25 & 0.547 & $1.202^{* *}$ & 972 & 180 & $1.25^{* *}$ & $121.813^{* *}$ & $136.281^{* *}$ & 0.001 \\
\hline GCA & 5 & $4.313^{*}$ & 1.163 & $3.084 * *$ & $1027.2 * *$ & 318.4 & $3.065^{* *}$ & $28.25 * *$ & $15.275^{* *}$ & $5.733 * *$ \\
\hline SCA & 15 & 1.241 & 1.975 & $1.543 * *$ & 286.93 & 512.53 & $1.395^{* *}$ & $8.159 * *$ & $7.685^{* *}$ & $1.089 * *$ \\
\hline Error & 40 & 2.626 & 7.14 & 0.207 & 606.8 & 1852.4 & 0.031 & 2.003 & 2.684 & 0.04 \\
\hline $\mathrm{GCA} / \mathrm{SCA}$ & & 3.475 & 0.589 & 1.999 & 3.58 & 0.621 & 2.197 & 3.462 & 1.988 & 5.264 \\
\hline
\end{tabular}


Cont.Table 4a:

\begin{tabular}{|c|c|c|c|c|c|c|c|c|c|}
\hline \multirow{3}{*}{ S.O.V } & \multicolumn{3}{|c|}{ Maturity } & \multirow{2}{*}{\multicolumn{3}{|c|}{ Grain filling period /day }} & \multirow{2}{*}{\multicolumn{3}{|c|}{ Grain filling rate/ day(g) }} \\
\hline & \multicolumn{3}{|c|}{ Thermal units } & & & & & & \\
\hline & Stress & Normal & Red\% & Stress & Normal & Red\% & Stress & Normal & Red\% \\
\hline Rep & 4128 & 17440.0 & 0.046 & 1.477 & 2.777 & 0.124 & 0.006 & 0.006 & 0.077 \\
\hline Genotypes & $14654.4 * *$ & 29003.2 & $7.612^{* *}$ & $24.267 * *$ & $22.397 *$ & $98.187^{* *}$ & $0.158 * *$ & $0.238^{* *}$ & $82.384 * *$ \\
\hline $\begin{array}{l}\text { Parent } \\
\text { (P) }\end{array}$ & $36099.2^{* *}$ & $36321.6^{*}$ & $9.065^{* *}$ & $60.189 * *$ & $44.989 * *$ & $74.778 * *$ & $0.234 * *$ & $0.325^{* *}$ & $23.823 * *$ \\
\hline $\begin{array}{l}\text { Crosses } \\
\text { (c) }\end{array}$ & $4546.3^{* *}$ & 20009.1 & $7.591 * *$ & $6.365^{*}$ & 8.555 & $119.575^{* *}$ & $0.095^{* *}$ & $0.163^{* *}$ & $109.172^{* *}$ \\
\hline prsc & $48944 * *$ & $118328 * *$ & $0.635^{* *}$ & $95.287 * *$ & $103.219 * *$ & $50.796 * *$ & $0.656 * *$ & $0.854 * *$ & 0.154 \\
\hline GCA & $10236.8^{* *}$ & 8012.8 & $5.957^{* *}$ & $13.677^{* *}$ & $13.683^{* *}$ & $24.359 * *$ & $0.056 * *$ & $0.102^{* *}$ & $25.638 * *$ \\
\hline SCA & $3100.8^{* *}$ & 10217.6 & $1.397^{* *}$ & $6.226 * *$ & 5.393 & $35.519 * *$ & $0.051 * *$ & $0.072 * *$ & $28.069 * *$ \\
\hline Error & 636 & 17178 & 0.033 & 2.792 & 11.228 & 0.159 & 0.016 & 0.023 & 0.096 \\
\hline GCA/SCA & 3.301 & 0.784 & 4.264 & 2.197 & 2.537 & 0.686 & 1.098 & 1.417 & 0.913 \\
\hline
\end{tabular}


Table 4b: Mean squares analysis for yield and its components under water stress and normal irrigated conditions

\begin{tabular}{|c|c|c|c|c|c|c|c|c|c|c|c|c|c|}
\hline \multirow{2}{*}{ S.O.V } & \multirow{2}{*}{ d.f } & \multicolumn{3}{|c|}{ No. of spikes/ plant } & \multicolumn{3}{|c|}{ No. of kernels / spike } & \multicolumn{3}{|c|}{ 100-kernel weight (g.) } & \multicolumn{3}{|c|}{ Grain yield./plant (g.) } \\
\hline & & Stress & Normal & SI & Stress & Normal & SI & Stress & Normal & SI & Stress & Normal & SI \\
\hline Rep & 2 & 16.01 & 17.88 & 0.00 & 79.33 & 142.89 & 0.006 & 0.606 & 0.472 & 0.136 & 17.813 & 25.88 & 0.001 \\
\hline Genotypes & 20 & $24.22 *$ & $23.67^{*}$ & $0.245^{* *}$ & $86.35^{*}$ & $91.42 *$ & $7.58 * *$ & $0.56 * *$ & $0.51^{* *}$ & $55.18^{* *}$ & $245.57^{* *}$ & $302.24 * *$ & $0.056 * *$ \\
\hline $\begin{array}{l}\text { Parents } \\
\text { (P) }\end{array}$ & 5 & $51.3^{* *}$ & 59.13 & $0.172 * *$ & $104.78^{*}$ & $85.62^{*}$ & $7.857^{* *}$ & $0.556^{* *}$ & $0.472^{* *}$ & $87.53^{* *}$ & $650.57 * *$ & $756.33 * *$ & $0.114 * *$ \\
\hline $\begin{array}{c}\text { Crosses } \\
\text { (c) }\end{array}$ & 14 & 15.88 & 12.26 & $0.289 * *$ & $84.86 *$ & $93.84 *$ & $6.457 * *$ & $0.531^{* *}$ & $0.505^{* *}$ & $46.109 * *$ & $103.247 * *$ & $143.258^{* *}$ & $0.036 * *$ \\
\hline prsc & 1 & 5.46 & 5.99 & 0.002 & 15.03 & $86.59 *$ & $21.986 * *$ & $0.983^{* *}$ & $0.763 * *$ & $20.439 * *$ & $213.063 * *$ & $257.6^{* *}$ & $0.043 * *$ \\
\hline GCA & 5 & $20.09 * *$ & $12.72^{* *}$ & $0.109 * *$ & $37.813^{*}$ & $43.02 *$ & $0.769 * *$ & $0.115^{* *}$ & $0.119 *$ & $19.097 * *$ & $161.788^{* *}$ & $190.789 * *$ & $0.059 * *$ \\
\hline SCA & 15 & 4.07 & 3.28 & $0.073 * *$ & 25.77 & 26.29 & $3.114 * *$ & $0.211^{*}$ & $0.187 * *$ & $18.159 * *$ & $55.212^{* *}$ & 70.733** & $0.005 * *$ \\
\hline Error & 40 & 10.18 & 11.84 & 0.003 & 42.88 & 42.82 & 0.008 & 0.165 & 0.158 & 0.04 & 13.986 & 15.263 & 0.002 \\
\hline GCA/SCA & & 4.936 & 3.878 & 1.493 & 1.467 & 1.636 & 0.247 & 0.545 & 0.636 & 1.052 & 2.93 & 2.697 & 11.8 \\
\hline
\end{tabular}

SI = susceptibility index 
Table 5. Mean performance $(x)$ for the earlines components and yield and its components under water stress and normal irrigated condition

\begin{tabular}{|c|c|c|c|c|c|c|c|}
\hline \multirow{3}{*}{\multicolumn{2}{|c|}{ Genotypes }} & \multicolumn{6}{|c|}{ Heading } \\
\hline & & \multicolumn{3}{|c|}{ Days } & \multicolumn{3}{|c|}{ Thermal units } \\
\hline & & Stress & Normal & Red\% & Stress & Normal & Red\% \\
\hline \multicolumn{2}{|c|}{$P_{1}$} & 90.00 & 98.67 & 87.90 & 1506.70 & 1638.00 & 8.02 \\
\hline \multicolumn{2}{|c|}{$\mathrm{P}_{2}$} & 92.00 & 100.33 & 8.30 & 1538.00 & 1666.80 & 7.73 \\
\hline \multicolumn{2}{|c|}{$P_{3}$} & 93.00 & 100.00 & 7.00 & 1553.00 & 1661.80 & 6.55 \\
\hline \multicolumn{2}{|c|}{$\mathrm{P}_{4}$} & 94.00 & 101.00 & 6.93 & 1568.00 & 1674.90 & 6.38 \\
\hline \multicolumn{2}{|c|}{$P_{5}$} & 92.00 & 103.00 & 10.68 & 1538.00 & 1708.70 & 9.99 \\
\hline \multicolumn{2}{|c|}{$P_{6}$} & 93.00 & 101.00 & 7.92 & 1553.00 & 1674.90 & 7.28 \\
\hline \multicolumn{2}{|c|}{$P_{1} \times P_{2}$} & 90.00 & 100.00 & 9.00 & 1506.70 & 1659.30 & 9.20 \\
\hline \multicolumn{2}{|c|}{$P_{1} \times P_{3}$} & 91.67 & 100.33 & 8.63 & 1532.40 & 1663.60 & 7.89 \\
\hline \multicolumn{2}{|c|}{$P_{1} \times P_{4}$} & 92.00 & 99.00 & 7.07 & 1538.00 & 1643.00 & 6.39 \\
\hline \multicolumn{2}{|c|}{$P_{1} \times P_{5}$} & 89.00 & 101.00 & 11.80 & 1491.10 & 1676.20 & 11.04 \\
\hline \multicolumn{2}{|c|}{$\mathrm{P}_{1} \times \mathrm{P}_{6}$} & 92.00 & 101.67 & 9.51 & 1538.00 & 1687.40 & 8.85 \\
\hline \multicolumn{2}{|c|}{$\mathrm{P}_{2} \times \mathrm{P}_{3}$} & 92.00 & 101.00 & 8.91 & 1538.00 & 1674.90 & 8.17 \\
\hline \multicolumn{2}{|c|}{$\mathrm{P}_{2} \times \mathrm{P}_{4}$} & 91.00 & 101.00 & 9.90 & 1523.00 & 1675.50 & 9.10 \\
\hline \multicolumn{2}{|c|}{$P_{2} \times P_{5}$} & 90.00 & 99.00 & 9.09 & 1506.70 & 1644.30 & 8.37 \\
\hline \multicolumn{2}{|c|}{$\mathrm{P}_{2} \times \mathrm{P}_{6}$} & 93.00 & 101.67 & 8.53 & 1553.00 & 1688.1 & 8.00 \\
\hline \multicolumn{2}{|c|}{$P_{3} \times P_{4}$} & 92.00 & 98.00 & 6.12 & 1538.00 & 1628 & 5.53 \\
\hline \multicolumn{2}{|c|}{$P_{3} X P_{5}$} & 91.00 & 100.00 & 9.00 & 1523.00 & 1659.9 & 6.25 \\
\hline \multicolumn{2}{|c|}{$P_{3} \times P_{6}$} & 93.00 & 102.00 & 8.82 & 1553.00 & 1693.10 & 8.27 \\
\hline \multicolumn{2}{|c|}{$\mathrm{P}_{4} \times \mathrm{P}_{5}$} & 95.00 & 102.67 & 7.47 & 1583.00 & 1703.10 & 7.05 \\
\hline \multicolumn{2}{|c|}{$\mathrm{P}_{4} \times \mathrm{P}_{6}$} & 92.33 & 100.67 & 8.28 & 1543.00 & 1669.30 & 7.57 \\
\hline \multicolumn{2}{|c|}{$P_{5} \times P_{6}$} & 92.33 & 89.67 & 6.43 & 1543.00 & 1639.30 & 5.87 \\
\hline \multirow{2}{*}{ L.S.D } & $5 \%$ & 2.59 & 4.28 & 0.73 & 39.42 & 68.88 & 0.28 \\
\hline & $1 \%$ & 3.55 & 5.85 & 1.00 & 53.90 & 94.18 & 0.38 \\
\hline
\end{tabular}


Table 5. Cont

\begin{tabular}{|c|c|c|c|c|c|}
\hline \multicolumn{6}{|c|}{ Maturity } \\
\hline \multicolumn{3}{|c|}{ Days } & \multicolumn{3}{|c|}{ Thermal unites } \\
\hline Stress & Normal & Red\% & Stress & Normal & Red\% \\
\hline 124.00 & 136.00 & 8.82 & 2064.2 & 2292.2 & 9.95 \\
\hline 127.00 & 136.00 & 6.62 & 2114.6 & 2292.2 & 7.75 \\
\hline 129.00 & 137.00 & 5.84 & 2148.2 & 2313.4 & 7.14 \\
\hline 139.33 & 145.67 & 4.35 & 2362.9 & 2515.8 & 5.74 \\
\hline 135.00 & 146.00 & 7.53 & 2271.0 & 2514.4 & 9.68 \\
\hline 130.00 & 137.00 & 5.11 & 2167.9 & 2313.4 & 10.14 \\
\hline 126.00 & 138.00 & 8.70 & 2097.8 & 2334.6 & 9.04 \\
\hline 126.00 & 136.67 & 7.81 & 2097.8 & 2306.3 & 10.05 \\
\hline 125.00 & 137.00 & 8.76 & 2081.0 & 2313.4 & 9.11 \\
\hline 124.00 & 135.00 & 8.15 & 2064.2 & 2271.0 & 5.26 \\
\hline 128.00 & 135.00 & 4.48 & 2131.4 & 2249.8 & 7.02 \\
\hline 128.00 & 136.00 & 5.88 & 2131.4 & 2292.2 & 7.94 \\
\hline 129.00 & 138.00 & 6.52 & 2149.7 & 2001.8 & 9.21 \\
\hline 125.00 & 136.00 & 8.09 & 2081.0 & 2292.2 & 6.44 \\
\hline 128.00 & 135.33 & 5.42 & 213.4 & 2278.1 & 6.89 \\
\hline 127.00 & 135.00 & 5.93 & 2114.6 & 2271.0 & 5.73 \\
\hline 129.67 & 136.00 & 4.65 & 2160.9 & 2292.2 & 6.92 \\
\hline 130.00 & 137.67 & 5.57 & 2166.5 & 2327.5 & 6.32 \\
\hline 132.33 & 139.33 & 5.02 & 2214.5 & 2364.0 & 6.15 \\
\hline 128.00 & 135.00 & 5.19 & 2131.40 & 2271.0 & 6.82 \\
\hline 128.67 & 136.33 & 5.62 & 2142.6 & 2299.3 & \\
\hline 2.26 & 2.62 & 0.32 & 40.35 & 209.7 & 0.29 \\
\hline 3.1 & 3.59 & 0.44 & 55.18 & 286.8 & 0.41 \\
\hline
\end{tabular}


Table 5. cont

\begin{tabular}{|c|c|c|c|c|c|}
\hline \multicolumn{3}{|c|}{ Grain filling period } & \multicolumn{3}{|c|}{ Grain filling rate } \\
\hline Stress & Normal & Red\% & Stress & Normal & Red\% \\
\hline 34.00 & 37.33 & 8.92 & 0.759 & 0.823 & 7.78 \\
\hline 36.33 & 35.67 & -1.85 & 1.150 & 1.315 & 12.55 \\
\hline 36.00 & 37.00 & 2.70 & 1.107 & 1.277 & 13.31 \\
\hline 45.33 & 44.67 & -1.48 & 1.197 & 1.384 & 13.51 \\
\hline 43.00 & 43.00 & 0.00 & 1.611 & 1.802 & 10.60 \\
\hline 37.00 & 36.00 & 2.70 & 1.326 & 1.579 & 16.02 \\
\hline 36.00 & 38.00 & 5.26 & 1.416 & 1.519 & 6.78 \\
\hline 34.33 & 36.33 & 5.51 & 1.190 & 1.290 & 7.75 \\
\hline 33.00 & 38.00 & 13.16 & 1.681 & 1.699 & 1.06 \\
\hline 35.00 & 34.00 & -2.94 & 1.436 & 1.712 & 16.12 \\
\hline 36.00 & 32.33 & -11.35 & 1.580 & 2.057 & 23.19 \\
\hline 36.00 & 35.00 & -2.86 & 1.177 & 1.357 & 13.26 \\
\hline 38.00 & 37.00 & -2.70 & 1.083 & 1.259 & 13.98 \\
\hline 35.00 & 37.00 & 5.41 & 1.469 & 1.523 & 3.55 \\
\hline 35.00 & 33.67 & -3.95 & 1.611 & 1.890 & 14.76 \\
\hline 35.00 & 37.00 & 5.41 & 1.520 & 1.639 & 7.26 \\
\hline 38.67 & 36.00 & -7.42 & 1.497 & 1.831 & 18.24 \\
\hline 37.00 & 35.67 & -3.73 & 1.231 & 1.480 & 16.82 \\
\hline 37.33 & 36.67 & -1.80 & 1.390 & 1.639 & 15.19 \\
\hline 35.67 & 37.33 & -3.90 & 1.598 & 1.898 & 15.81 \\
\hline 36.33 & 37.67 & 3.56 & 1.383 & 1.520 & 9.01 \\
\hline 2.67 & 5.36 & 0.64 & 0.204 & 0.245 & 0.496 \\
\hline 3.66 & 7.33 & 0.87 & 0.278 & 0.335 & 0.678 \\
\hline
\end{tabular}


Table 5. cont

\begin{tabular}{|c|c|c|c|c|c|}
\hline \multicolumn{3}{|c|}{ No. of spike / plant } & \multicolumn{3}{|c|}{ No. of kernels / spike } \\
\hline Stress & Normal & S.I & Stress & Normal & S.I \\
\hline 14.42 & 16.89 & 1.367 & 69.00 & 68.94 & -0.054 \\
\hline 14.92 & 16.22 & 0.756 & 61.33 & 62.11 & 0.785 \\
\hline 17.45 & 19.25 & 0.882 & 61.47 & 60.64 & -0.855 \\
\hline 20.39 & 22.60 & 0.922 & 68.39 & 67.75 & -0.590 \\
\hline 25.20 & 28.00 & 0.943 & 77.33 & 75.17 & -1.796 \\
\hline 15.70 & 18.14 & 1.269 & 66.69 & 69.89 & 2.862 \\
\hline 17.78 & 20.22 & 1.138 & 77.00 & 77.78 & 0.627 \\
\hline 16.00 & 18.22 & 1.149 & 69.11 & 71.61 & 2.182 \\
\hline 18.83 & 20.72 & 0.861 & 72.72 & 73.35 & 0.537 \\
\hline 19.32 & 21.92 & 1.119 & 66.27 & 66.12 & -0.142 \\
\hline 19.14 & 20.83 & 0.765 & 67.25 & 78.75 & 1.984 \\
\hline 17.55 & 19.41 & 0.904 & 69.73 & 70.49 & 0.674 \\
\hline 17.42 & 18.75 & 0.669 & 59.58 & 62.09 & 2.527 \\
\hline 17.63 & 20.44 & 1.297 & 61.38 & 64.11 & 2.661 \\
\hline 17.08 & 19.58 & 1.205 & 66.50 & 64.01 & -2.431 \\
\hline 21.78 & 23.84 & 0.815 & 65.08 & 67.6 & 2.330 \\
\hline 23.75 & 25.03 & 0.482 & 65.47 & 66.33 & 0.810 \\
\hline 18.67 & 20.86 & 0.990 & 63.41 & 64.17 & 0.740 \\
\hline 22.11 & 24.00 & 0.743 & 68.42 & 72.00 & 3.108 \\
\hline 17.42 & 20.25 & 1.318 & 75.99 & 78.33 & 1.867 \\
\hline 15.47 & 18.89 & 1.708 & 69.89 & 73.44 & 3.021 \\
\hline 5.107 & 2.508 & 0.093 & 10.48 & 10.47 & 0.139 \\
\hline 6.982 & 7.531 & 0.127 & 14.33 & 14.32 & 0.190 \\
\hline
\end{tabular}

S.I. Susceptibility index 
Table 5. cont

\begin{tabular}{|c|c|c|c|c|c|}
\hline \multicolumn{3}{|c|}{100 -graim weight } & \multicolumn{3}{|c|}{ Grain yield / plant } \\
\hline Stress & Normal & S.I & Stress & Normal & S.I \\
\hline 4.78 & 4.88 & 3.82 & 25.85 & 30.63 & 1.264 \\
\hline 4.97 & 4.99 & 0.746 & 41.4 & 46.24 & 0.848 \\
\hline 5.34 & 5.66 & 10.528 & 39.72 & 47.07 & 1.265 \\
\hline 5.89 & 5.89 & 0.00 & 54.55 & 61.81 & 0.951 \\
\hline 5.76 & 5.58 & -6.01 & 69.2 & 77.24 & 0.843 \\
\hline 5.39 & 5.46 & 2.387 & 48.96 & 56.79 & 1.117 \\
\hline 5.82 & 5.79 & -0.965 & 50.91 & 57.61 & 0.942 \\
\hline 5.7 & 5.68 & -0.656 & 40.69 & 46.84 & 1.064 \\
\hline 5.92 & 5.9 & -0.631 & 55.11 & 64.62 & 1.192 \\
\hline 5.93 & 5.9 & -0.947 & 50.15 & 57.67 & 1.056 \\
\hline 5.89 & 5.94 & 1.568 & 56.97 & 66.45 & 1.156 \\
\hline 5.72 & 5.66 & -1.974 & 42.25 & 47.45 & 0.888 \\
\hline 5.42 & 5.46 & 1.364 & 41.1 & 46.22 & 0.897 \\
\hline 5.58 & 5.33 & -8.725 & 50.94 & 56.35 & 0.778 \\
\hline 5.84 & 5.85 & 0.318 & 56.48 & 63.37 & 0.881 \\
\hline 5.47 & 5.6 & 4.323 & 53.25 & 60.47 & 0.967 \\
\hline 5.01 & 5.16 & 5.413 & 57.83 & 65.55 & 0.954 \\
\hline 4.92 & 4.87 & -1.912 & 45.48 & 52.45 & 1.075 \\
\hline 5.05 & 5.19 & 5.023 & 51.88 & 59.31 & 1.015 \\
\hline 6.56 & 6.56 & 0.00 & 56.98 & 65.16 & 1.017 \\
\hline 5.66 & 5.9 & 7.575 & 50.24 & 57.1 & 0.973 \\
\hline 0.65 & 0.64 & 0.322 & 5.99 & 6.252 & 0.066 \\
\hline 0.889 & 0.87 & 0.439 & 8.18 & 8.549 & 0.090 \\
\hline
\end{tabular}


Table 6. Estimate of general combining ability effects for the earliness components and yield and its components under water stress and normal irrigated conditions.

\begin{tabular}{|c|c|c|c|c|c|c|c|c|c|c|c|c|c|c|c|c|c|c|c|}
\hline \multirow{3}{*}{\multicolumn{2}{|c|}{ Genotype }} & \multicolumn{6}{|c|}{ Heading } & \multicolumn{6}{|c|}{ Maturity } & \multirow{2}{*}{\multicolumn{3}{|c|}{ Grain felling period }} & \multirow{2}{*}{\multicolumn{3}{|c|}{ Grain felling rate / day(g) }} \\
\hline & & \multicolumn{3}{|c|}{ Daye } & \multicolumn{3}{|c|}{ Thermal units } & \multicolumn{3}{|c|}{ Daye } & \multicolumn{3}{|c|}{ Thermal unites } & & & & & & \\
\hline & & Stress & Normal & Red\% & Stress & Normal & Red\% & Stress & Normal & Red\% & Stress & Normal & Red\% & Stress & Normal & Red\% & Stress & Normal & Red\% \\
\hline \multicolumn{2}{|c|}{$\mathrm{g} 1$} & $-1.097 * *$ & -0.528 & $0.531^{* *}$ & $\begin{array}{c}- \\
16.989 * *\end{array}$ & -8.893 & $0.531 * *$ & $-2.833^{* *}$ & $-1.042 * *$ & $1.357 * *$ & $\begin{array}{c}- \\
50.961^{* *}\end{array}$ & -9.467 & $1.288^{* *}$ & $-1.792 * *$ & -0.639 & $3.075 * *$ & $-0.081 * *$ & $-0.114 * *$ & $-1.882^{* *}$ \\
\hline \multicolumn{2}{|c|}{ g2 } & -0.431 & -0.028 & $0.326 * *$ & -6.511 & -0.127 & $0.392 * *$ & $-1.208^{* *}$ & $-0.708^{*}$ & $0.395 * *$ & $\begin{array}{c}- \\
23.478^{* *}\end{array}$ & -43.996 & $0.382^{* *}$ & -0.5 & -0.806 & $-0.678 * *$ & $-0.052 *$ & $-0.082 * *$ & $-1.011^{* *}$ \\
\hline \multicolumn{2}{|c|}{ g3 } & 0.278 & -0.278 & $\begin{array}{c}- \\
0.493 * *\end{array}$ & 4.351 & -4.193 & $\begin{array}{c}- \\
0.495 * *\end{array}$ & -0.125 & $-0.708^{*}$ & $\begin{array}{c}- \\
0.396 * *\end{array}$ & -5.095 & -2.4 & $\begin{array}{c}- \\
0.415^{* *}\end{array}$ & -0.458 & -0.556 & -0.072 & $-0.08^{* *}$ & $-0.085 * *$ & $0.554 * *$ \\
\hline \multicolumn{2}{|c|}{ g4 } & $0.861^{*}$ & -0.028 & $-0.84 * *$ & $13.181^{* *}$ & -0.997 & $\begin{array}{c}- \\
0.846^{* *}\end{array}$ & $2.542^{* *}$ & $1.833^{* *}$ & $\begin{array}{c}- \\
0.571^{* *}\end{array}$ & $51.072^{* *}$ & 14.687 & $\begin{array}{c}- \\
0.546^{* *}\end{array}$ & $1.625^{* *}$ & $2.111^{* *}$ & $0.541^{* *}$ & 0.025 & 0.009 & $-0.65 * *$ \\
\hline \multicolumn{2}{|c|}{ g5 } & -0.264 & 0.472 & $0.726 * *$ & -4.09 & 7.928 & $0.674 * *$ & $1.25 * *$ & $1.708^{* *}$ & $0.238^{* *}$ & $24.605 * *$ & $\begin{array}{c}51.525 \\
*\end{array}$ & $0.419 * *$ & $1.458^{* *}$ & 1.111 & $-0.759 * *$ & $0.116^{* *}$ & $0.125 * *$ & $-0.276 * *$ \\
\hline \multicolumn{2}{|c|}{ g6 } & $0.653 *$ & 0.389 & $\begin{array}{c}- \\
0.251^{* *}\end{array}$ & $10.056^{*}$ & 6.282 & $\begin{array}{c}- \\
0.256 * *\end{array}$ & 0.375 & $-1.083 * *$ & $\begin{array}{c}- \\
1.023^{* *}\end{array}$ & 3.855 & 10.35 & -1.128 & -0.333 & $-1.222^{*}$ & $-2.107 * *$ & $0.073^{* *}$ & $0.146 * *$ & $3.254 * *$ \\
\hline \multirow{2}{*}{$\begin{array}{l}\text { L. } \\
\text { S. } \\
\text { D } \\
\mathrm{g}_{i}\end{array}$} & \begin{tabular}{|l|}
5 \\
$\%$ \\
\end{tabular} & 0.619 & 1.021 & 0.174 & 9.41 & 16.44 & 0.067 & 0.54 & 0.626 & 0.076 & 9.634 & 50.07 & 0.069 & 0.638 & 1.28 & 0.152 & 0.048 & 0.057 & 0.118 \\
\hline & $\begin{array}{l}1 \\
\%\end{array}$ & 0.833 & 1.374 & 0.234 & 12.67 & 22.14 & 0.091 & 0.728 & 0.843 & 0.103 & 12.97 & 67.41 & 0.093 & 0.859 & 1.72 & 0.205 & 0.065 & 0.078 & 0.159 \\
\hline \multirow{2}{*}{$\begin{array}{l}\text { L. } \\
\text { S. } \\
\text { D } \\
g_{11} \\
-g_{i}\end{array}$} & $\begin{array}{l}5 \\
\%\end{array}$ & 0.959 & 1.25 & 0.269 & 14.578 & 25.47 & 0.104 & 0.838 & 0.97 & 0.118 & 14.524 & 77.56 & 0.108 & 0.989 & 1.98 & 0.235 & 0.075 & 0.09 & 0.183 \\
\hline & $\begin{array}{c}1 \\
\%\end{array}$ & 1.291 & 2.13 & 0.362 & 19.626 & 34.29 & 0.14 & 1.128 & 1.305 & 0.159 & 20.093 & 104.4 & 0.145 & 1.331 & 2.66 & 0.318 & 0.101 & 0.121 & 0.247 \\
\hline
\end{tabular}


Table 6. Cont.

\begin{tabular}{|c|c|c|c|c|c|c|c|c|c|c|c|}
\hline \multicolumn{3}{|c|}{ No. of spikes / plant } & \multicolumn{3}{|c|}{ No. of kernels / spike } & \multicolumn{3}{|c|}{ 100-Kernel Weight $(\mathrm{g})$} & \multicolumn{3}{|c|}{ Grain yield /plant $(\mathrm{g})$} \\
\hline Stress & Normal & S.I & Stress & Normal & S.I & Stress & Normal & S.I & Stress & Normal & S.I \\
\hline-1.180 & -1.126 & $0.081^{* *}$ & $2.793^{*}$ & $2.575^{*}$ & $-0.234 * *$ & -0.006 & -0.014 & $-0.134 * *$ & $-5.138 * *$ & $-5.126 * *$ & $0.111^{* *}$ \\
\hline$-1.506 *$ & $-1.729 *$ & $-0.047 * *$ & $-2.518^{*}$ & $-2.775 *$ & $-0.165^{* *}$ & -0.07 & -0.126 & $-1.947 * *$ & $-2.772 * *$ & $-4.0^{* *}$ & $-0.121^{* *}$ \\
\hline 0.413 & 0.147 & $-0.125^{* *}$ & $-2.658^{*}$ & $-2.926 *$ & $-0.24 * *$ & $-0.171^{* *}$ & -0.1 & $2.397^{* *}$ & $-3.463 * *$ & $-3.57 * *$ & $0.054 * *$ \\
\hline 1.126 & 1.009 & $-0.106 * *$ & 0.196 & 0.497 & $0.28 * *$ & $0.165^{* *}$ & $0.175^{*}$ & $0.375^{* *}$ & $2.595^{* *}$ & $2.992^{* *}$ & -0.007 \\
\hline $2.417^{* *}$ & $2.699 *$ & 0.017 & 1.135 & 0.93 & $-0.135^{* *}$ & -0.016 & -0.056 & $-1.346^{* *}$ & $6.6^{* *}$ & $6.874 * *$ & $-0.073 * *$ \\
\hline-1.270 & $-0.999 *$ & $0.178^{* *}$ & 1.052 & 1.699 & $0.495 * *$ & 0.097 & 0.012 & $0.656 * *$ & $2.177^{* *}$ & $2.831^{* *}$ & $0.036 * *$ \\
\hline 1.219 & 1.314 & 0.021 & 2.501 & 2.5 & 0.034 & 0.155 & 0.152 & 0.076 & 1.429 & 1.492 & 0.016 \\
\hline 1.641 & 1.77 & 0.028 & 3.368 & 3.365 & 0.045 & 0.209 & 0.204 & 0.103 & 1.923 & 2.009 & 0.021 \\
\hline 1.888 & 2.036 & 0.032 & 3.875 & 3.872 & 0.052 & 0.24 & 0.235 & 0.118 & 2.213 & 2.312 & 0.024 \\
\hline 2.542 & 2.742 & 0.044 & 5.217 & 5.214 & 0.07 & 0.324 & 0.317 & 0.159 & 2.98 & 3.113 & 0.033 \\
\hline
\end{tabular}


Estimates of GCA effects ( $\hat{g}_{i}$ ) for individual parents to each trait in stress and non stress experiments as well as thermal units, reduction and drought susceptibility index are presented in Table (6). Highly significant negative $\left(\hat{g}_{i}\right)$ values would be of interest for most traits of heading and maturity (days and thermal units) and susceptibility index, whereas highly significant and positive $\left(\hat{g}_{i}\right)$ values are preferred for grain yield and its components and the reduction percentage under both experiments.

Under stress condition, parent $P_{1}$ ranked the best combiner for heading and maturity (days and thermal units), grain filling period and number of kernels / spike, while $\mathrm{P}_{4}$ ranked the second best general combiner for grain yield / plant and the first combiner for kernel weight. Parent $P_{5}$ expressed the highest significant $\left(\hat{g}_{i}\right)$ effects for grain yield / plant and number of spikes / plant, and $\mathrm{P}_{6}$ ranked the third best general combiner for grain yield.

In normal experiments condition, parent P1 ranked the second best combiner for number of days of maturity (early) and the first best combiner of number kernels / spike, parents $\mathrm{P}_{2}$ and $\mathrm{P}_{3}$ the third best combiner for number of days of maturity, while parent $\mathrm{P}_{4}$ ranked the best combiner for kernel weight and the second best general combiner. Parent $p_{5}$ expressed the most desirable $\left(\hat{g}_{i}\right)$ effects for number of spikes / plant and grain yield / plant, wheals parent P 6 the best desirable ( $\left.\hat{g}_{i}\right)$ effects for number of days for maturity (early), the best of grain filling rate and the third best combiner of grain yield / plant.

For the reduction percentage, $\mathrm{P}_{1}$ was the second best combiner for heading (days and thermal units), the first best for maturity (days and thermal units) and the best for grain filling pried and filling rate while $\mathrm{P}_{2}$ was the second best general combiner for heading (days and thermal units) and the second for maturity. $\mathrm{P}_{4}$ consider the second combiner for grain filling period. While $P_{5}$ ranked the first best combiner for heading (days and maturity)

For drought susceptibility index, parent $\mathrm{P}_{1}$ was the best combiner for number of kernels / spike and kernel weight, parent $P_{2}$ considers a good combiner for grain yield and its components, while $\mathrm{P}_{3}$ ranked the first best combiner for number of spikes / plant and number of kernels / spike. Parent $\mathrm{P}_{4}$ ranked the second best combiner for number of spikes / plant, whears $\mathrm{P}_{5}$ considered a good combiner for number of kernels / spike, kernel weight and grain yield / plant.

Specific combining ability effects for all the studied traits in stress and non stress conditions, thermal units, reduction and susceptibility index are presented in Table (7). In stress condition, the most desirable Sij effects were detected by the cross combinations $\mathrm{P}_{1} \times \mathrm{P}_{4}$ for early maturity ( days and thermal units ) and grain filling 
rate, $\mathrm{P}_{1} \times \mathrm{P}_{5}$ for early heading and maturity ( days and thermal units ), $\mathrm{P}_{2} \times \mathrm{P}_{5}$ and $\mathrm{P}$ ${ }_{3} \times \mathrm{P}_{4}$ for early maturity ( days and thermal units), $\mathrm{P}_{1} \times \mathrm{P}_{2}$ for number of kernels / spike and grain yield, $\mathrm{P}_{1} \times \mathrm{P}_{4}$ for grain yield / plant, $\mathrm{P}_{1} \times \mathrm{P}_{6}$ for number of spikes / plant and grain yield, $\mathrm{P}_{2} \times \mathrm{P}_{6}, \mathrm{P}_{3} \times \mathrm{P}_{4}$ and $\mathrm{P}_{3} \times \mathrm{P}_{5}$ for grain yield, plant.

Under normal condition, specific combining ability effects were detected in the crosses; $\mathrm{P}_{1} \times \mathrm{P}_{5}$ and $\mathrm{P}_{2} \times \mathrm{P}_{5}$ for early maturity of number of day, $\mathrm{P}_{3} \times \mathrm{P}_{4}, \mathrm{P}_{3} \times \mathrm{P}_{5}$ and $\mathrm{P}_{4} \times \mathrm{P}_{5}$ for early maturity number of days and grain filling rate and $\mathrm{P}_{5} \times \mathrm{P}_{6}$ for early heading of number days, $\mathrm{P}_{1} \times \mathrm{P}_{2}$ for number of kernels / spike and grain yield / plant, $\mathrm{P}_{1} \times \mathrm{P}_{4}, \mathrm{P}_{2} \times \mathrm{P}_{6}, \mathrm{P}_{3} \times \mathrm{P}_{4}$ and $\mathrm{P}_{3} \times \mathrm{P}_{5}$ for grain yield / plant and $\mathrm{P}_{1} \times \mathrm{P}_{6}$ for number of spikes / plant and grain yield / plant.

Regarding reduction percentage, six, eight, eight, eight, six and nine crosses expressed significant and positive Sij effects for number of days for heading, thermal units for heading, number of days for maturity, thermal units for maturity, grain filling period and grain filling rate, respectively. 
Table 7. Estimate of specific combining ability effects for the earliness components and yield and its components under water stress and normal irrigated conditions

\begin{tabular}{|c|c|c|c|c|c|c|c|c|c|c|c|c|c|c|c|c|c|c|c|}
\hline \multirow{3}{*}{\multicolumn{2}{|c|}{ Genotype }} & \multicolumn{6}{|c|}{ Heading } & \multicolumn{6}{|c|}{ Maturity } & \multirow{2}{*}{\multicolumn{3}{|c|}{ Grain felling period }} & \multirow{2}{*}{\multicolumn{3}{|c|}{ Grain felling rate / day $(g)$}} \\
\hline & & \multicolumn{3}{|c|}{ Daye } & \multicolumn{3}{|c|}{ Thermal units } & \multicolumn{3}{|c|}{ Daye } & \multicolumn{3}{|c|}{ Thermal units } & & & & & & \\
\hline & & Stress & Normal & Red\% & Stress & Normal & Red\% & Stress & Normal & Red\% & Stress & Normal & Red\% & Stress & Normal & Red\% & Stress & Normal & Red\% \\
\hline \multicolumn{2}{|c|}{$\mathrm{P}_{1} \times \mathrm{P}_{2}$} & -0.393 & 0.048 & -0.346 & -6.277 & 0.194 & $0.397 * *$ & $1.518^{*}$ & $2.464 * *$ & $0.564 * *$ & $28.198^{*}$ & $83 . .025$ & $0.871^{* *}$ & $1.625 *$ & 2.524 & $2.451 * *$ & $0.196 * *$ & $0.166 *$ & $-2.544 * *$ \\
\hline \multicolumn{2}{|c|}{$\mathrm{P}_{1 \times P_{3}}$} & 0.565 & 0.631 & 0.103 & 8.493 & 8.627 & -0.027 & 0.435 & 1.131 & $0.465 * *$ & 9.815 & 13.162 & $0.567 * *$ & -0.083 & 0.607 & $2.095 * *$ & -0.001 & -0.059 & $-3.139 * *$ \\
\hline \multicolumn{2}{|c|}{$P_{1} \times P_{4}$} & 0.315 & -0.952 & $-1.11 * *$ & 5.297 & -15.202 & $-1.176 * *$ & $-3.23 * *$ & -1.077 & $1.59 * *$ & $-63.15^{* *}$ & 3.141 & $1.708 * *$ & $-3.5 * *$ & -0.393 & $9.133 * *$ & $0.385 * *$ & $0.257 * *$ & $-8.625 * *$ \\
\hline \multicolumn{2}{|c|}{$P_{1} \times P_{5}$} & -1.26 & 0.548 & $2.134 * *$ & $-24.332^{*}$ & 9.04 & $1.957 * *$ & $-2.94 * *$ & $-2.952 * *$ & 0.172 & $-53.49 * *$ & -76.096 & $-0.197 * *$ & -1.333 & $-3.393 *$ & $-5.667 * *$ & 0.049 & $0.153 *$ & $6.061 * *$ \\
\hline \multicolumn{2}{|c|}{$P_{1} \times P_{6}$} & 0.524 & 1.298 & $0.741 * *$ & 8.422 & 21.952 & $0.694 * *$ & $1.935 * *$ & -1.161 & $-2.237 * *$ & $34.46 * *$ & -35.421 & $-2.499 * *$ & $1.458^{*}$ & -2.726 & $-12.73 * *$ & $0.235 * *$ & $0.477 * *$ & $9.591 * *$ \\
\hline \multicolumn{2}{|c|}{$\mathrm{P}_{2} \times \mathrm{P}_{3}$} & 0.232 & 0.798 & $0.588^{* *}$ & 3.652 & 11.128 & $0.394 * *$ & 0.18 & 0.131 & $-0.503 * *$ & 15.931 & 33.558 & $-0.547 * *$ & 0.292 & -0.56 & $-2.522 * *$ & -0.044 & -0.023 & $1.5^{* *}$ \\
\hline \multicolumn{2}{|c|}{$P_{2} \times P_{4}$} & -1.351 & 0.548 & $1.925 * *$ & -20.177 & 8.565 & $1.673^{* *}$ & -0.857 & -0.411 & $0.312^{* *}$ & $-21.969 *$ & $-273.9 * *$ & $0.505 * *$ & 0.208 & -1.226 & $-2.975 * *$ & $-0.243 * *$ & $-0.215 * *$ & $3.424 * *$ \\
\hline \multicolumn{2}{|c|}{$P_{2 \times P} P_{5}$} & -1.226 & -1.952 & $-0.451^{*}$ & -19.173 & -31.627 & $-0.577 * *$ & $-3.57 * *$ & $-2.286 * *$ & $1.073 * *$ & $-64.17 * *$ & -20.37 & $0.81 * *$ & $-2.625 * *$ & -0.226 & $6.435 * *$ & 0.052 & -0.067 & $-7.38 * *$ \\
\hline \multicolumn{2}{|c|}{$P_{2} \times P_{6}$} & 0.857 & 0.798 & -0.034 & 12.948 & 13.819 & -0.017 & 0.31 & -0.161 & $-0.336 * *$ & 6.981 & 27.38 & $-0.431 * *$ & -0.833 & -1.226 & $-1.577 * *$ & $0.237 * *$ & $0.277 * *$ & $0.29 *$ \\
\hline \multicolumn{2}{|c|}{$P_{3} \times P_{4}$} & -1.06 & -2.202 & $-1.036 * *$ & -16.04 & -34.902 & $-1.01 * *$ & $-3.94 * *$ & $-3.411 * *$ & $0.513 * *$ & $-75.42 * *$ & -46.33 & $0.251 * *$ & $-2.833 * *$ & -1.476 & $4.529 * *$ & $0.223 * *$ & $0.168 *$ & $-4.861 * *$ \\
\hline \multicolumn{2}{|c|}{$P_{3} \times P_{5}$} & -0.935 & -0.702 & 0.278 & -13.769 & -11.927 & $0.189 *$ & 0.018 & $-2.286 * *$ & $-1.576 * *$ & -2.686 & -61.96 & $-1.874 * *$ & 1.0 & -1.476 & $-7.001^{* *}$ & 0.108 & $0.244 * *$ & $5.745 * *$ \\
\hline \multicolumn{2}{|c|}{$P_{3} \times P_{6}$} & 0.149 & 1.381 & $1.075^{* *}$ & 2.085 & 22.886 & 1. $14 * *$ & $1.226 * *$ & $2.173 * *$ & $0.605 * *$ & $23.664 *$ & 35.25 & $0.863 * *$ & 1.125 & 0.524 & $-1.964 * *$ & $-0.115 * *$ & -0.129 & $0.785 * *$ \\
\hline \multicolumn{2}{|c|}{ P4XPs } & $2.482^{* *}$ & 1.714 & $-0.905 * *$ & $37.402 * *$ & 28.044 & $-0.66 * *$ & 0.018 & $-1.494 *$ & $-1.031^{* *}$ & -5.252 & -7.25 & $-1.153^{* *}$ & $-2.417^{* *}$ & $-3.476 *$ & $-1.994 * *$ & -1.104 & -0.042 & $3.899 * *$ \\
\hline \multicolumn{2}{|c|}{$P_{4} \times P_{6}$} & -1.101 & -0.202 & $0.883^{* *}$ & -16.744 & -4.11 & $0.791^{* *}$ & $-3.44 * *$ & $-3.036 * *$ & $0.4 * *$ & $-67.57 * *$ & -38.38 & $0.225 * *$ & $-2.292 * *$ & -0.476 & $-2.746 * *$ & $0.147 *$ & $0.195 * *$ & $0.979 * *$ \\
\hline \multicolumn{2}{|c|}{$P_{5 \times} P_{6}$} & 0.024 & $-2.702^{*}$ & $-2.534 * *$ & 0.527 & $-43.035 *$ & $-2.43 * *$ & $-1.482^{*}$ & $-1.577^{*}$ & 0.022 & $-29.90 * *$ & -46.95 & -0.07 & $-1.458^{*}$ & 0.857 & $6.014 * *$ & $-0.159 * *$ & $-0.298 * *$ & $-6.195 * *$ \\
\hline \multirow{2}{*}{$\begin{array}{c}\text { L.S.D } \\
\text { sij }\end{array}$} & $5 \%$ & 1.403 & 2.315 & 0.394 & 21.34 & 37.28 & 0.153 & 1.226 & 1.419 & 0.173 & 21.85 & 113.5 & 0.157 & 1.447 & 2.903 & 0.345 & 0.11 & 0.131 & 0.268 \\
\hline & $1 \%$ & 1.89 & 3.12 & 0.531 & 28.73 & 50.2 & 0.205 & 1.65 & 1.911 & 0.233 & 29.41 & 152.9 & 0.212 & 1.949 & 3.908 & 0.465 & 0.148 & 0.177 & 0.361 \\
\hline \multirow{2}{*}{$\begin{array}{l}\text { L.S.D } \\
\text { sij-sik }\end{array}$} & $5 \%$ & 2.54 & 4.18 & 0.712 & 38.57 & 67.39 & 0.277 & 2.22 & 2.57 & 0.313 & 39.49 & 205.2 & 0.28 & 2.616 & 5.25 & 0.624 & 0.198 & 0.237 & 0.485 \\
\hline & $1 \%$ & 3.42 & 5.63 & 0.959 & 51.93 & 90.73 & 0.371 & 2.98 & 3.45 & 0.422 & 53.16 & 276.3 & 0.38 & 3.52 & 7.06 & 0.841 & 0.267 & 0.32 & 0.653 \\
\hline \multirow{2}{*}{$\begin{array}{l}\text { L.S.D } \\
\text { sij-sk| }\end{array}$} & $5 \%$ & 2.35 & 3.87 & 0.66 & 35.7 & 62.39 & 0.255 & 2.05 & 2.37 & 0.29 & 36.56 & 190 & 0.263 & 2.422 & 4.86 & 0.5780 & 0.183 & 0.22 & 0.449 \\
\hline & $1 \%$ & 3.16 & 5.21 & 0.888 & 48.07 & 83.99 & 0.343 & 2.76 & 3.2 & 0.39 & 49.22 & 255.8 & 0.354 & 3.261 & 6.45 & 0.778 & 0.247 & 0.296 & 0.605 \\
\hline
\end{tabular}


Table 7. Cont:

\begin{tabular}{|c|c|c|c|c|c|c|c|c|c|c|c|}
\hline \multicolumn{3}{|c|}{ No. of spikes / plant } & \multicolumn{3}{|c|}{ No. of kernels / spike } & \multicolumn{3}{|c|}{ 100-Kernel Weight (g) } & \multicolumn{3}{|c|}{ Grain yield /plant (g.) } \\
\hline Stress & Normal & S.I & Stress & Normal & S.I & Stress & Normal & S.I & Stress & Normal & S.I \\
\hline 1.989 & 2.409 & $0.087 * *$ & $8.582 * *$ & $8.706 * *$ & 0.033 & 0.344 & $0.35^{*}$ & 0.105 & $9.302 * *$ & $10.244 * *$ & $-0.055^{* *}$ \\
\hline-1.71 & -1.471 & $0.176^{* *}$ & 0.831 & 2.691 & $1.663 * *$ & 0.325 & 0.207 & $-3.93^{* *}$ & -0.227 & -0.956 & $-0.108 * *$ \\
\hline 0.411 & 0.164 & $-0.13^{* *}$ & 1.591 & 1.008 & $-0.502 * *$ & 0.204 & 0.152 & $-1.884 * *$ & $8.131^{* *}$ & $10.262 * *$ & $0.081 * *$ \\
\hline-0.398 & -0.326 & $0.005^{*}$ & $-5.906 *$ & $-6.659 *$ & $-0.766 * *$ & $0.396 *$ & $0.389 *$ & $-0.478 * *$ & -0.83 & -0.577 & 0.011 \\
\hline $3.109 *$ & 2.288 & $-0.51 * *$ & 4.261 & 5.206 & $0.73 * *$ & 0.244 & 0.253 & 0.035 & $10.409 * *$ & $12.253 * *$ & 0.002 \\
\hline 0.166 & 0.323 & $0.061 *$ & $6.765^{*}$ & $6.924 *$ & $0.087 *$ & $0.405^{*}$ & 0.306 & $-3.434 * *$ & -1.037 & -1.475 & $-0.052 * *$ \\
\hline-0.68 & -1.196 & $-0.192 * *$ & $-6.238^{*}$ & -4.906 & $1.419 * *$ & -0.226 & -0.172 & $1.925^{* *}$ & $-8.242 * *$ & $-9.271 * *$ & 0.018 \\
\hline-1.761 & -1.196 & $0.313 * *$ & -5.381 & -3.316 & $1.969 * *$ & 0.113 & -0.072 & $-6.453 * *$ & -2.41 & -3.02 & -0.035 \\
\hline 1.382 & 1.642 & $0.06 *$ & -0.178 & -4.185 & $-3.753 * *$ & 0.257 & 0.276 & $0.599 * *$ & $7.55^{* *}$ & $8.04 * *$ & $-0.041 *$ \\
\hline 1.768 & 2.019 & 0.032 & -0.599 & 0.759 & $1.298^{* *}$ & -0.078 & -0.062 & $0.54 * *$ & $4.593 * *$ & $4.55^{*}$ & $-0.086 * *$ \\
\hline 2.493 & 1.512 & $-0.425^{* *}$ & -1.156 & -0.941 & $0.193 * *$ & $-0.352^{*}$ & -0.265 & $3.352^{* *}$ & $5.175^{* *}$ & $5.747 * *$ & -0.033 \\
\hline 1.046 & 1.043 & $-0.078^{* *}$ & -3.129 & -3.876 & $-0.507 * *$ & $-0.561 * *$ & $-0.731^{* *}$ & $-5.975 * *$ & -2.759 & -3.303 & -0.021 \\
\hline 0.09 & -0.377 & $-0.182^{* *}$ & -1.052 & 1.302 & $1.971^{* *}$ & $-0.65 * *$ & $-0.517 * *$ & $4.983 * *$ & $-6.834 * *$ & $-7.048^{* *}$ & $0.089 * *$ \\
\hline-0.913 & -0.429 & $0.232 * *$ & $6.598^{*}$ & $6.867 *$ & $0.1^{*}$ & $0.741^{* *}$ & $0.681^{* *}$ & $-2.042 * *$ & 2.682 & 2.838 & -0.019 \\
\hline - 4.151** & $-3.479 * *$ & $0.499 * *$ & -0.442 & 1.544 & $1.669 * *$ & 0.023 & 0.248 & $7.259 * *$ & $-8.056 * *$ & $-9.101 * *$ & 0.003 \\
\hline 2.764 & 2.981 & 0.047 & 5.673 & 5.669 & 0.077 & 0.352 & 0.344 & 0.173 & 3.24 & 3.384 & 0.036 \\
\hline 3.721 & 4.013 & 0.064 & 7.637 & 7.632 & 0.103 & 0.474 & 0.464 & 0.233 & 4.362 & 4.556 & 0.048 \\
\hline 4.996 & 5.388 & 0.086 & 10.253 & 10.246 & 0.138 & 0.636 & 0.622 & 0.313 & 5.855 & 6.117 & 0.065 \\
\hline 6.726 & 7.253 & 0.115 & 13.804 & 13.794 & 0.186 & 0.856 & 0.838 & 0.422 & 7.883 & 8.235 & 0.087 \\
\hline 4.625 & 4.988 & 0.079 & 9.492 & 9.486 & 0.128 & 0.589 & 0.576 & 0.29 & 5.421 & 5.663 & 0.06 \\
\hline 6.227 & 6.715 & 0.107 & 12.78 & 12.771 & 0.172 & 0.793 & 0.776 & 0.39 & 7.299 & 7.625 & 0.08 \\
\hline
\end{tabular}


Whears draught susceptibility index, different crosses expressed significant and negative Sij effects for number of spikes / plant, (in six crosse) number kernels / spikes, kernel weight (in seven crosses) and grain yield / plant (in five crosses) respectively.

In conclusion, parents $\mathrm{P}_{1}$ and $\mathrm{P}_{2}$ are the best combiners for early maturing, parents $\mathrm{P}_{4}$ and $\mathrm{P}_{5}$ could be considered as good combiners for grain yield and most of its components and parent $\mathrm{P}_{6}$ considered the best combiner for early mature and grain yield / plant under stress and non stress experiments.

It could be concluded that hybrid $\mathrm{P}_{1} \times \mathrm{P}_{4}$ and $\mathrm{P}_{3} \times \mathrm{P}_{4}$ seem to be the best combination among studied crosses as it expressed the most desirable Sij effects for early maturity (days and thermal units) and high grain yield / plant under stress condition and for drought susceptibility index while $\mathrm{P}_{3} \times \mathrm{P}_{4}$ and $\mathrm{P}_{3} \times \mathrm{P}_{5}$ expressed the most desirable Sij effects for early maturity (days and thermal units) under normal condition. Therefore, it may be prospective in wheat breeding programs towards the development of new genotypes characterized by higher yield potentiality, early maturity and resistance to drought condition.

\section{REFERENCES}

1. Abdel- Nour, Nadya A.R. 2005. Genetic Studies for yield and its components on drought and drought susceptibility index in wheat. Egypt. J.Agric.Res. 83 (4).

2. Abdel- Nour, Nadya A.R. and Manal A. Hassam. 2009. Determination of gene effects and variance in three bread wheat crosses for low water (drought). Egypt J. plant Breed 13:235-249.

3. Abul - Naas, A.A, Sh.A. El- Shamarka; A.A. El- Hosary and I. H. Darwish. 2000. Genetical studies on drought susceptibility index for yield and its components in wheat. J. Agric. Sci. Mansoura Univ., 25(12): 7457-7472.

4. Ammar, S.El.M.M. 2003. Estimates of genetic variance for yield and its components in wheat under normal and drought conditions. Egypt.J.plant Breed. 7(2):93-110.

5. Fischer, R.A. and R. Maurer. 1978. Drought resistance in spring wheat cultivars.I.Crain yield response. Aust. I.Agric.Res.29:897-912.

6. Clarke, J.M., T.F.C.Townle y, T.N.C.Mc and D.G.Green 1984. Growth analysis of spring wheatcultivars of varying drought resistene. Crop Sc.24:537-541. 
7. Griffing J.B. 1956. Concept of general and specific combining ability in relation to diallel crossing systems. Australian J.Biol.,Sci., 9:463- 493.

8. Gomez- Macpherson, H. and R.A.Richards. 1997. Effect of early sowing on development in wheat isoclines differing in verbalization and photoperiod requirements. Field Crops Research 54:91-107.

9. Kato, K. and H. Yokoyama. 1992. Geographical variation in heading characters among wheat landraces Triticum L.and its implication for their adaptability. Theor. Appl. Genet.84:259-265.

10. halif, M. A. ,A. A. Ismaiel,G.R. El- Nagar and I. M. Amin. 1998. Genetical studies of earliness, grain yield and its components of bread wheat. Assiut J.Agric.Sci., 29(5):59-69.

11. Masle, J, G. Doussinault and B. Sun. 1989. Response of wheat genotypes to temperature and photo period in natural condition. Crop. Sci., 29:712-721.

12. Menshawy, A.M.M. 2005. Genetic analysis for earliness components in some wheat genotypes of different photo thermal response. Fourth plant Breeding Conference (March 5). Egypt. J.plant Breed . 8(1):31-37. Special Issue.

13. Menshawy, A.M.M. 2007. Evaluation of some ealy bread wheat genotypes under different sowing dates: 1-Earliness characters, 2Agronomic characters. Fifth plant Breeding Conference (May 27 Giza). Egypt.J.plant Breed. 11(1): 25-55.

14. Steel, R.G.D.and J. H. Torrie. 1980. Principles and procedures of statisties. A biometrical approach. Second Ed. McGraw-Hill PP. 167-173. 


\title{
دراسات وراثية لتحمل المحصول للجفاف والتبكير فى النضج لأقماح الديورم

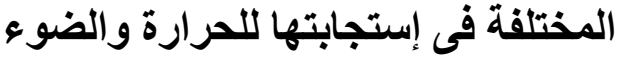

\author{
نادية عدلى رياض عبد النور1 ، هيام سيد أحمد فاتح2 \\ 1. قسم بحوث القهح - معهز المحاصيل الحقلية ـ مركز البحوث الزراعية

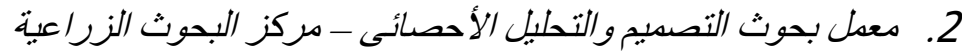

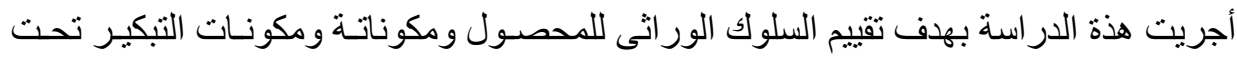

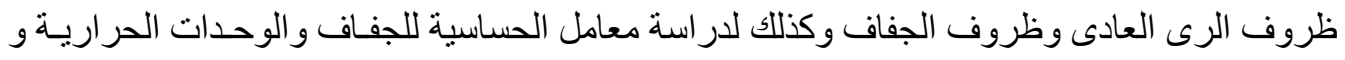

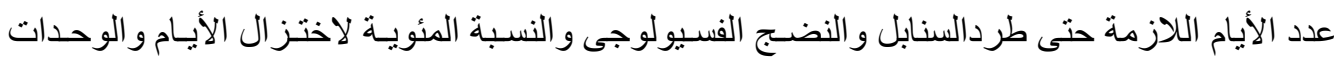

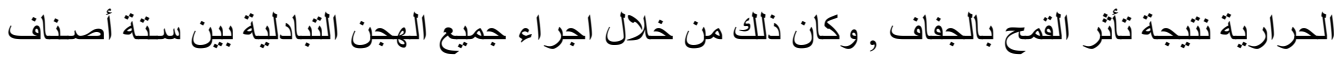

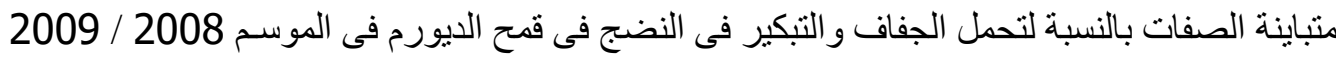

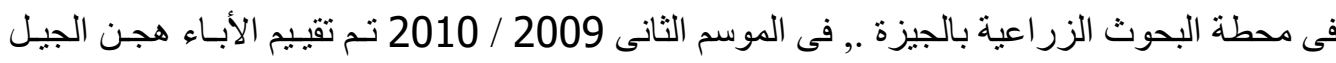
الأول F1 فى محطة البحوث الزر اعية بسدس وذللك بزر اعتها فى تجربتين، الأولى تم ريها مـرة و احلدة

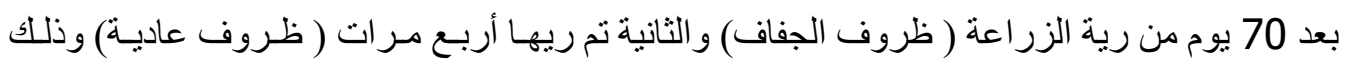

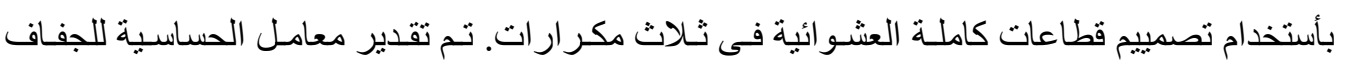

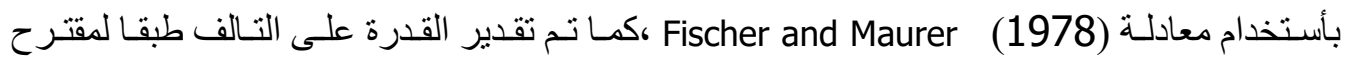
Griffing (1956) الطريقة الثانية النموذج الأول وقد تم حساب عدد الايام و الوحدات الحرارية حتى طرد

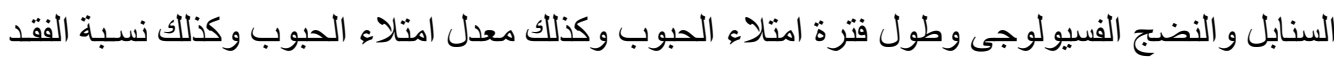

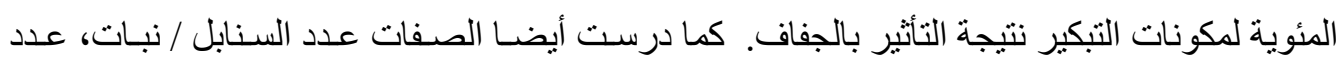

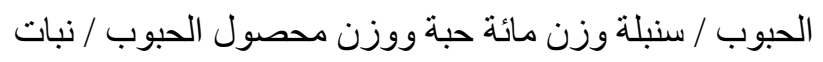

\section{وكانت أهم النتائج هى : - الت}

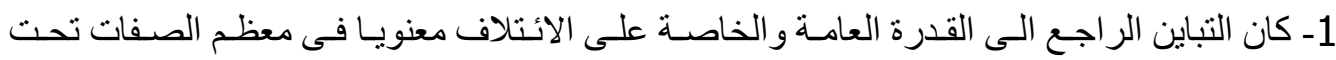

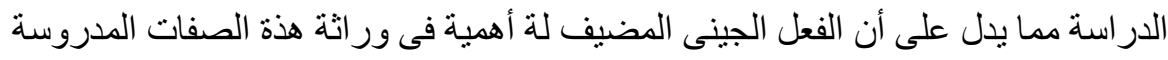

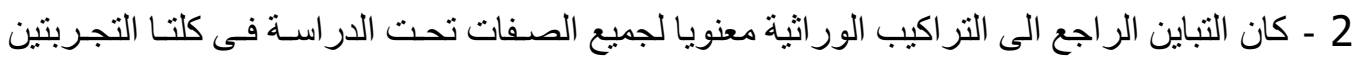

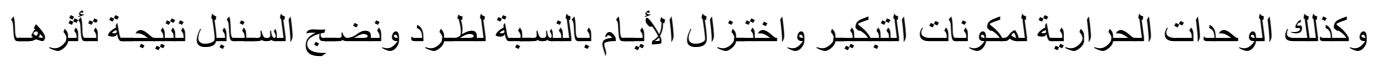

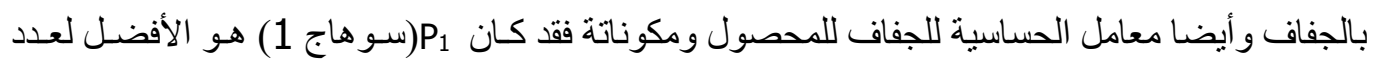

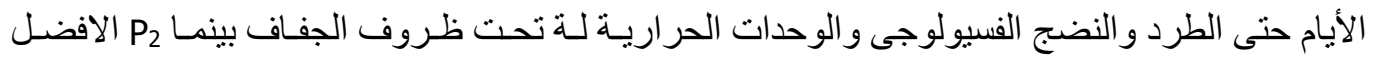

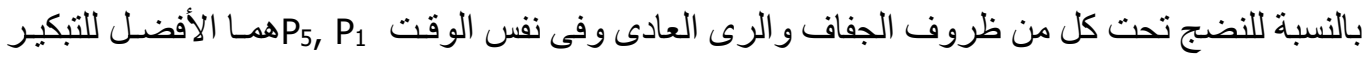

$$
\text { فى النضج تحت ظروف الرى العادى. }
$$

قد كان P5 هو الافضل بالنسبة لعدد السنابل / نبات ووزن محصول النبات بالنسبة لظروف الجفاف و الرى الرى

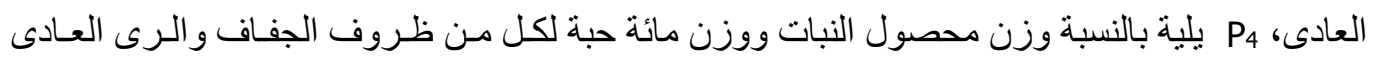

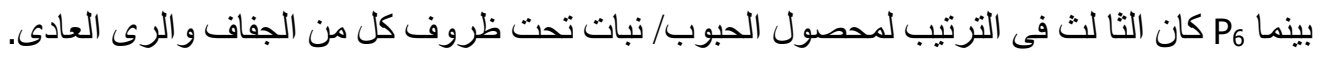

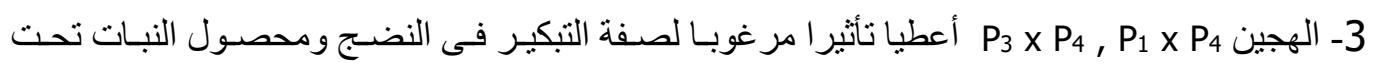

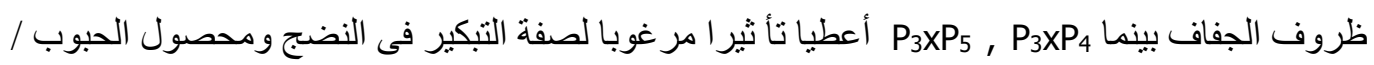

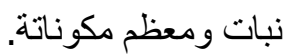




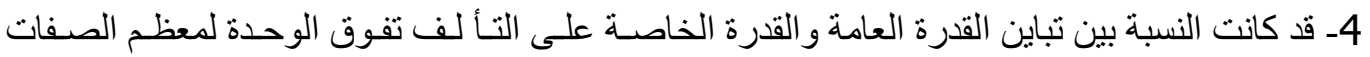

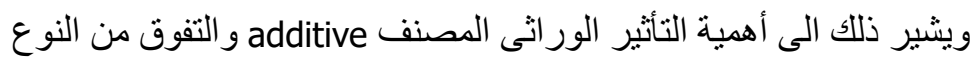

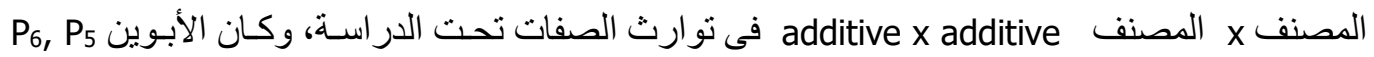

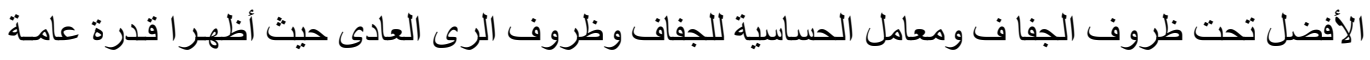
مر غوبة لصفة محصول الحبوب النبات ومعظم مكوناتة. 